\title{
Effect of Mutual Coupling on Multiple Antenna Channel
}

\author{
Xue Cuiwei, Zhu Qiuming, Chen Xiaomin, Liu Xinglin, and Yang Ying
}

\begin{abstract}
The influence mechanism of mutual coupling (MC) on multiple antennas system are studied. Some new exact expressions of receiving signal vector, signal power and spatial correlation coefficient are derived, which includes the effect of MC. The capacity of multiple-input multiple- output (MIMO) channel with MC in different conditions is also discussed. Analysis and simulation results show that the modification of signal parameters cased by MC is related to the MC matrix, which is decided by antenna self-impendence, mutual impendence and load impedance.
\end{abstract}

Index Terms-Mutual coupling (MC), MIMO channel, antenna pattern, channel capacity.

\section{INTRODUCTION}

Multiple-input multiple-output (MIMO) technology can significantly improve the channel capacity of wireless systems under rich propagation environment. However, the advantage of MIMO system over signal antenna system depends on the channel characteristics such as spatial correlation. Since antenna's feathers are inherently included in the communication link, mutual coupling not only affects the antenna efficiency but also the system performance [1].

Ref. [2] proved that the distance between antennas and the operation frequency difference are the main affect factors of mutual coupling. Wang in [3] presented an iterative autocalibration method to estimate unknown mutual coupling for a uniform circular array. In [4], the network theory was used to derive the channel transfer matrix, which includes the coupling effects on spatial correlation between different antennas. Lu and Hui [5] also proved that MC among antennas would limit the increase of capacity due to spatial proximity. In [6], the effects of MC on MIMO channel capacity under different coupling assumptions were analyzed and compared. [7] presented a comparison between the measured mutual coupling effect on the ergodic channel capacity of a MIMO system in a Rayleigh channel and in Rician channel at Long Term Evolution (LTE) radio band. A method to reduce MC among MIMO antennas by inserting parasitic elements is proposed in [8], and the 1-D EBG and SRR structures were experimentally shown to be very effective in suppressing mutual coupling [9].

In this paper, we will study the MC effect on multiple antennas system and derive some new expressions of receiving signal vector and power, channel correlation and capacity. Firstly, we construct an equivalent channel model

Manuscript received May 10, 2016; revised July 13, 2016.

The authors are with the Jiangsu Key Laboratory of Internet of Things and Control Technologies, Nanjing University of Aeronautics and Astronautics, Nanjing 211100, China. Zhu Qiuming is also with China Airborne Missile Academy, Luoyang 471009, China (e-mail: zhuqiuming@nuaa.edu.cn). with $\mathrm{MC}$ in both the transmitting and receiving arrays and give the receiving signal model including coupling in Section II. On this basis, the signal power and spatial correlation between multiple receiving antennas including MC will be given, and the effect of MC on channel capacity will also be studied in combining with cross-correlation and total power in Section III. Finally, some conclusions will be drawn in Section IV.

\section{System Model with Mutual COUPLing}

The MC describes the electromagnetic interaction between multiple antenna elements. When antenna elements are very close, the field generated by one antenna will alter the current distributions of others. In this way, the radiation pattern and input impedance of each element are disturbed by the presence of the other elements, which is similar with signal crosstalk in circuit system. The equivalent MIMO system $\left(N_{t} \times N_{r}\right)$ model with MC is shown in Fig. 1.

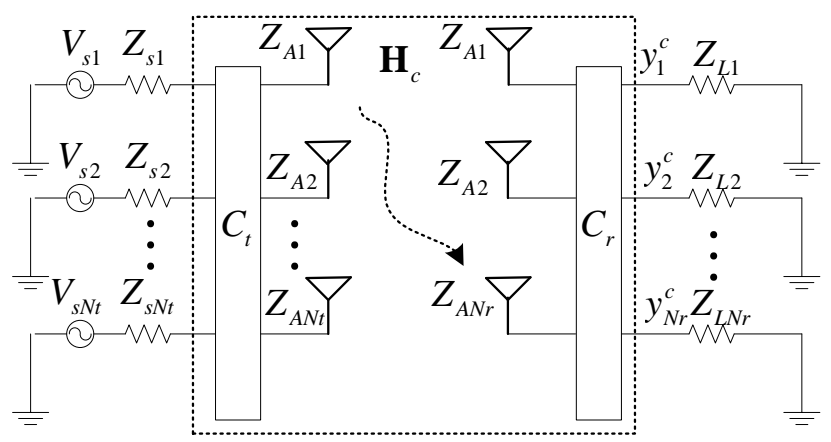

Fig. 1. System model with mutual coupling.

We assume that the elements of antenna arrays are vertically polarized. The transmitting or receiving signal vector (radiation pattern) including MC should be revised as [10]

$$
\mathbf{y}_{t / r}^{c}(\phi)=\mathbf{C}_{t / r} \mathbf{y}_{t / r}^{n c}(\phi)
$$

where $\phi$ is the azimuth angle between departure or arrival wave direction with antenna array, $\mathbf{y}_{t / r}^{n c}(\phi)$ is transmitting or receiving signal vector without considering $\mathrm{MC}$, which can be written as

$$
\mathbf{y}_{t / r}^{n c}(\phi)=\mathbf{g}_{t / r}^{n c}(\phi) \odot \mathbf{a}_{t / r}(\phi)
$$

where $\mathbf{C}_{t / r}$ is the complex coupling matrix on transmitter or receiver, $\mathbf{g}_{t / r}^{n c}(\phi)$ and $\mathbf{a}_{t / r}(\phi)$ are the gain vector and steering vector of antenna array respectively.

Taking the receiver as an example, the following content will illustrate how the MC changes receiving signal's character. Firstly, the coupling matrix $\mathbf{C}_{r}$ can be calculated by [10]. 


$$
\mathbf{C}_{r}=\left(z_{L}+z_{A}\right)\left(\mathbf{Z}_{L}+\mathbf{Z}_{r}\right)^{-1}
$$

where $\mathbf{Z}_{r}$ is the mutual impedance matrix, $\mathbf{Z}_{L}$ is the load impendence matrix. $z_{A}$ and $z_{L}$ are the antenna impendence and load impedance, which have the same value on each branch for simplicity. For a side-by-side array, the expressions for $Z_{m n}^{r}$ are [11]

$$
Z_{m n}^{r}=\left\{\begin{array}{l}
30[0.577+\ln (2 \pi)-\operatorname{Ci}(2 \pi) \\
+j \operatorname{Si}(2 \pi)] \\
30\left[2 \operatorname{Ci}(\beta d)-\operatorname{Ci}\left(\beta \mu_{1}\right)-\operatorname{Ci}\left(\beta \mu_{2}\right)\right] \\
-30 j\left[2 \operatorname{Si}(\beta d)-\operatorname{Si}\left(\beta \mu_{1}\right)-\operatorname{Si}\left(\beta \mu_{2}\right)\right], m \neq n
\end{array}, m=n\right.
$$

where

$$
\left\{\begin{array}{c}
\beta=2 \pi / \lambda \\
\mu_{1}=\sqrt{d^{2}+L^{2}}+L \\
\mu_{2}=\sqrt{d^{2}+L^{2}}-L
\end{array}\right.
$$

$L$ is the length of the antenna, $d$ is the inter-element distance of antenna array, and $\operatorname{Ci}(x), \operatorname{Si}(x)$ are the cosine and sine integral respectively[12],

$$
\begin{aligned}
& C i(x)=\int_{-\infty}^{x} \frac{\cos x}{x} d x \\
& \operatorname{Si}(x)=\int_{-\infty}^{x} \frac{\sin x}{x} d x
\end{aligned}
$$

\section{Mutual Coupling EFFeCts}

\section{A. Signal Vector and Power}

Taking account of the MC, the signal vector can be rewritten with the help of circuit theory and (1)-(2) as

$$
\begin{aligned}
& \mathbf{y}^{c}(\phi)=\left[\begin{array}{llll}
y_{1}^{c}(\phi) & y_{2}^{c}(\phi) & \cdots & y_{N}^{c}(\phi)
\end{array}\right]^{T} \\
& =\left[\begin{array}{lll}
\sum_{n=1}^{N} C_{1 n} g_{n}^{n c}(\phi) a_{n}(\phi) & \cdots & \sum_{n=1}^{N} C_{N n} g_{n}^{n c}(\phi) a_{n}(\phi)
\end{array}\right]^{T}
\end{aligned}
$$

Here, the subscript $r$ is omitted for brevity. The new expression shows that the signal vector of each element is the weighted sum of all branches. For example, we assume two omnidirectional antennas array, so the original signal vector is given by

$$
\mathbf{y}^{n c}(\phi)=\left[\begin{array}{ll}
y_{1}^{n c}(\phi) & y_{2}^{n c}(\phi)
\end{array}\right]^{T}=\left[\begin{array}{ll}
1 & e^{j 2 \pi d \sin \phi / \lambda}
\end{array}\right]^{T}
$$

Here we assume two dipole antennas of $L=\lambda / 2$, placed side by side with $d=0.3 \lambda$, which is also assumed to be omnidirectional in order to facilitate the research. For such antennas, the self-impedance is $z_{A}=(73+42 j) \Omega$. When the load impedance matches to self-impendence $\left(z_{L}=z_{A}^{*}\right)$, the MC matrix can be calculated by (3) as

$$
\mathbf{C}_{r}=\left[\begin{array}{lr}
0.91-0.07 j & -0.09+0.30 j \\
-0.09+0.30 j & 0.91-0.07 j
\end{array}\right\rfloor
$$

Fig. 2 presents the magnitude of the signal vector, computed by (7) for different angle of incident wave. As we can see that the magnitude curves of antenna 1 and antenna 2 are the same, because the symmetry of MC matrix. Above all, the MC effect can be seen as a distortion of the signal vector or radiation pattern.

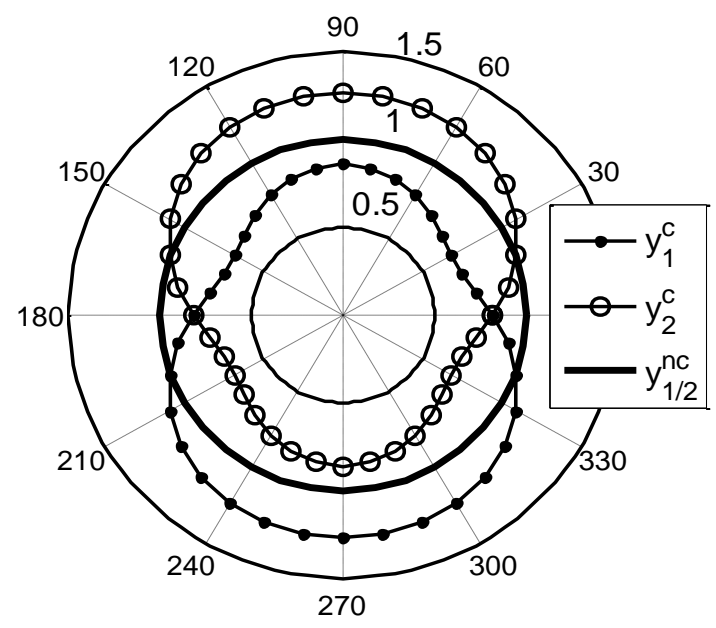

Fig. 2. Effect of MC on signal vector.

The signal power of each branch on the receiver without $\mathrm{MC}$, as well as on the transmitter, is defined by

$$
P_{k}^{n c}=\int_{\phi_{0}-\Delta}^{\phi_{0}+\Delta}\left|y_{k}^{n c}(\phi)\right|^{2} p(\phi) d \phi
$$

Assuming normalized signal power on each element $\left(P_{k}^{n c}=1\right)$, so the total receiving power denoted by $\hat{P}^{n c}$ equals $N$. Considering the effect of $\mathrm{MC}$, the new signal power of each branch can be derived from (7) and (10) as

$$
\begin{aligned}
P_{k}^{c}= & \int_{\phi_{0}-\Delta}^{\phi_{0}+\Delta}\left|y_{k}^{c}(\phi)\right|^{2} p(\phi) d \phi \\
= & \int_{\phi_{0}-\Delta}^{\phi_{0}+\Delta} \sum_{n=1}^{N} C_{k n} y_{n}^{n c}(\phi)\left(\sum_{m=1}^{N} C_{k m} y_{m}^{n c}(\phi)\right)^{*} p(\phi) d \phi \\
= & \int_{\phi_{0}-\Delta}^{\phi_{0}+\Delta}\left\{\sum_{n=1}^{N} \sum_{n=1}^{N} \sum_{m=1, m \neq n}^{N} C_{k n}^{*} y_{n}^{n c}(\phi)\left(y_{n}^{n c}(\phi)\right)^{*}+\right. \\
= & \left.\sum_{n=1}^{N} C_{k n}^{*} C_{k n}^{*} \int_{\phi_{0}-\Delta}^{\phi_{0}+\Delta} y_{n}^{n c}(\phi)\left(y_{m}^{n c}(\phi)\right)^{*}\right\} p(\phi) d \phi \\
& \sum_{n=1}^{N} \sum_{m=1, m \neq n}^{N} C_{k n} C_{k m}^{*} \int_{\phi_{0}-\Delta}^{\phi_{0}+\Delta} y_{n}^{n c}(\phi)\left(y_{m}^{n c}(\phi)\right)^{*} p(\phi) d \phi \\
= & \sum_{n=1}^{N} C_{k n} C_{k n}^{*}+\sum_{n=1}^{N} \sum_{m=1, m \neq n}^{N} C_{k n} C_{k m}^{*} \rho_{n, m}^{n c}
\end{aligned}
$$

where $\rho_{n, m}^{n c}$ is the correlation coefficient without MC, which is defined as

$$
\rho_{n, m}^{n c}=\frac{\int_{\phi_{0}-\Delta}^{\phi_{0}+\Delta} y_{n}^{n c}(\phi)\left(y_{m}^{n c}(\phi)\right)^{*} p(\phi) d \phi}{\sqrt{P_{n}^{n c} P_{m}^{n c}}}
$$

So, the sum of receiving signal power is

$$
\hat{P}^{c}=\sum_{k=1}^{N} P_{k}^{c}=\sum_{k=1}^{N}\left\{\sum_{n=1}^{N} C_{k n} C_{k n}^{*}+\sum_{n=1}^{N} \sum_{m=1, m \neq n}^{N} C_{k n} C_{k m}^{*} \rho_{n, m}^{n c}\right\}
$$

We assume the same parameters of antennas array as in Fig. 2 and the angle of arrival (AOA) has a Gaussian 
distribution with angle spread of $60^{\circ}$. Fig. 3 shows the receiving signal power loss due to $\mathrm{MC}$ with different load impedances. As we can see that the power loss is significant and decreases with the increase of inter-element distance, when $d / \lambda$ is less than 0.5 . If $d / \lambda$ is larger than 0.5 , the loss tends to be zero, and it fluctuates slightly between 0.97 and 1 . We also find that the power loss is almost same for different load impedances.

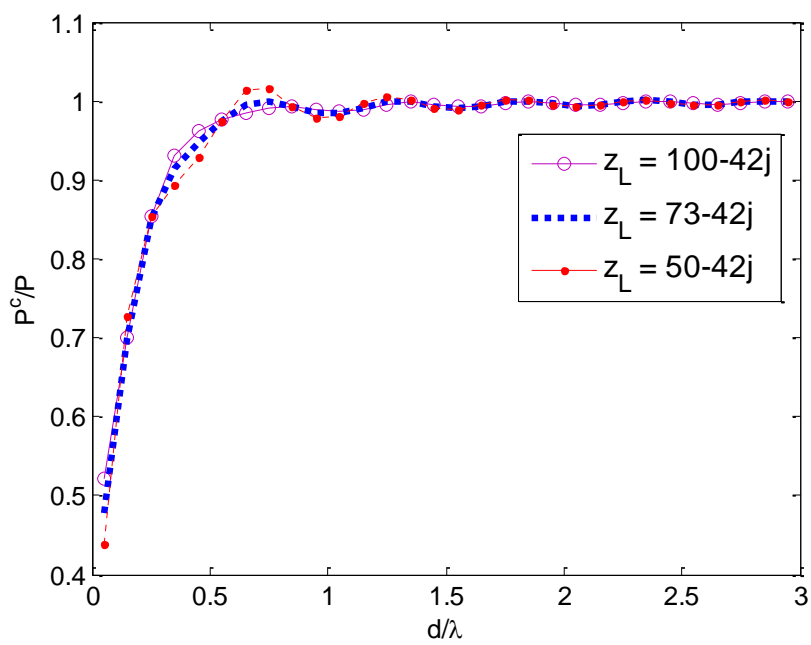

Fig. 3. Signal power loss due to MC.

\section{B. Spatial Correlation}

Using the definition of (12), the cross-correlation coefficient without MC can be derived as

$$
\rho_{n, m}^{n c}=\int_{\phi_{0}-\Delta}^{\phi_{0}+\Delta} e^{j 2 \pi(n-m) d \sin \phi / \lambda} p(\phi) d \phi
$$

Submitting (7) into (12) and making some derivations, the cross-correlation coefficient between antenna $k$ and $l$ with $\mathrm{MC}$ effects can be derived as

$$
\begin{aligned}
& \rho_{k, l}^{c}=\frac{1}{\sqrt{P_{k}^{c} P_{l}^{c}}} \int_{\phi_{0}-\Delta}^{\phi_{0}+\Delta} \sum_{n=1}^{N} C_{k n} y_{n}^{n c}(\phi)\left(\sum_{m=1}^{N} C_{l m} y_{m}^{n c}(\phi)\right)^{*} p(\phi) d \phi \\
& =\frac{1}{\sqrt{P_{k}^{c} P_{l}^{c}}}\left\{\begin{array}{l}
\int_{\phi_{0}-\Delta}^{\phi_{0}+\Delta} \sum_{n=1}^{N} C_{k n} C_{l n}^{*} y_{n}^{n c}(\phi)\left(y_{n}^{n c}(\phi)\right)^{*} p(\phi) d \phi+ \\
\int_{\phi_{0}-\Delta}^{\phi_{0}+\Delta} \sum_{n=1}^{N} \sum_{m=1, m \neq n}^{N} C_{k n} C_{l m}^{*} y_{n}^{n c}(\phi)\left(y_{m}^{n c}(\phi)\right)^{*} p(\phi) d \phi
\end{array}\right\} \\
& =\frac{1}{\sqrt{P_{k}^{c} P_{l}^{c}}}\left\{\begin{array}{l}
\sum_{n=1}^{N} C_{k n} C_{l n}^{*}+\sum_{n=1}^{N} \sum_{m=1, m \neq n}^{N} C_{k n} C_{l m}^{*} \rho_{n, m}^{n c}
\end{array}\right\}
\end{aligned}
$$

where $P_{k}^{c}, P_{l}^{c}, \rho_{n, m}^{n c}$ can be estimated by (11) and (14).

With the same simulation parameters as above, the MC effect on the absolute value of cross-correlation coefficient with different $d$ is shown in Fig.4. It can be found that all correlation coefficient curves decrease rapidly when interelement distance increases, and the existence of $\mathrm{MC}$ will reduce the correlation. Moreover, different load impedances have different effect, but they also tend to be same as $d / \lambda$ more than 2 .

\section{A. Channel Capacity}

Channel capacity is an important indicator of wireless MIMO system. According to (1), the new MIMO channel with MC can be rewritten as [13-14]

$$
\mathbf{H}^{c}=\mathbf{C}_{r} \mathbf{H}^{n c} \mathbf{C}_{t}
$$

where $\mathbf{H}^{n c}$ is the channel matrix without $\mathbf{M C}, \mathbf{C}_{r}$ and $\mathbf{C}_{t}$ are the $\mathrm{MC}$ matrices on receiver and transmitter. The ergodic capacity of MIMO channel, with the strategy of uniformly distributed transmitting power, is determined by

$$
\bar{C}=\mathrm{E}_{\mathbf{H}}\left\lfloor\log _{2} \operatorname{det}\left(\mathbf{I}_{N_{r}}+\frac{\zeta}{N_{t}} \mathbf{H}^{c}\left(\mathbf{H}^{c}\right)^{H}\right)\right\rfloor
$$

where $\xi$ is the signal-to-noise ratio,$N_{t}$ denotes the number of transmit antenna, the subscript ${ }^{H}$ denotes Hermitian transposition, and the expectation value is taken over all possible channel conditions described by $\mathbf{H}^{c}$.

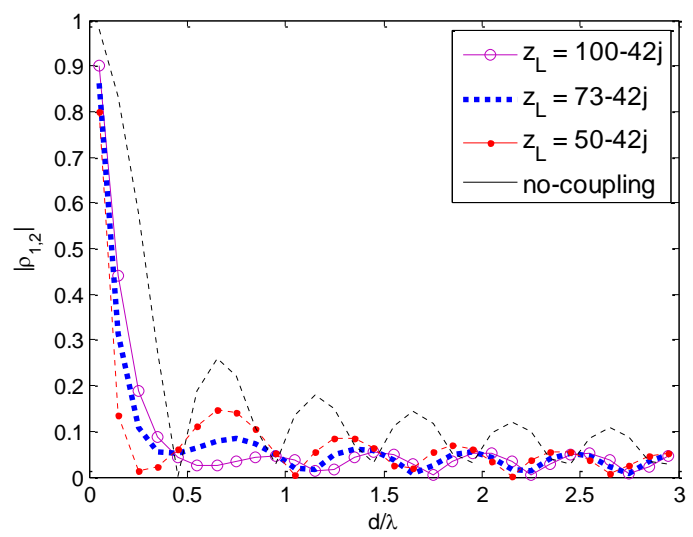

Fig. 4. Effect of mutual coupling on the correlation.

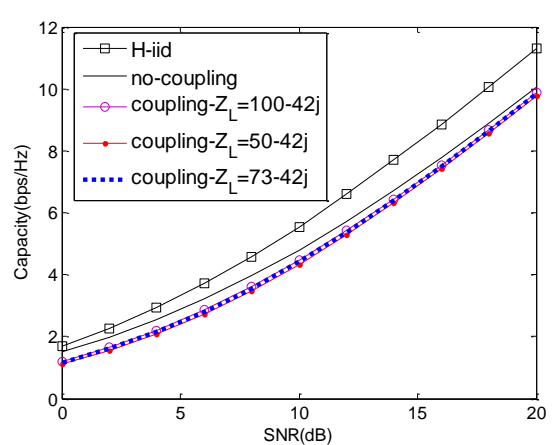

Fig. 5. Capacity of $2 \times 2$ MIMO system $(d / \lambda=0.3)$.

The covariance matrix of the channel can be expressed as $\boldsymbol{\rho}^{c}=\boldsymbol{\rho}_{t}^{c} \otimes \boldsymbol{\rho}_{r}^{c}$, where $\boldsymbol{\rho}_{t}^{c}$ and $\boldsymbol{\rho}_{r}^{c}$ are the covariance matrices of the transmitting and the receiving arrays respectively, and $\otimes$ represents a Kronecker product. Thus, the transfer matrix $\mathbf{H}^{c}$ can be reduced to

$$
\mathbf{H}^{c}=\left(\boldsymbol{\rho}_{r}^{c}\right)^{1 / 2} \mathbf{H}_{i i d}\left(\boldsymbol{\rho}_{t}^{c}\right)^{1 / 2}
$$

where $\mathbf{H}_{i i d}$ is the channel matrix generated using i.i.d. zeromean complex Gaussian random variables. Thus, $\bar{C}$ can be further expressed as

$$
\bar{C}=\mathrm{E}_{\mathbf{H}}\left[\log _{2} \operatorname{det}\left(\begin{array}{l}
\mathbf{I}_{N_{r}}+\frac{\zeta}{N_{t}}\left(\boldsymbol{\rho}_{r}^{c}\right)^{1 / 2} \cdot \mathbf{H}_{i i d} \cdot \\
\boldsymbol{\rho}_{t}^{c} \cdot\left(\mathbf{H}_{i i d}\right)^{H} \cdot\left(\left(\boldsymbol{\rho}_{r}^{c}\right)^{1 / 2}\right)^{H}
\end{array}\right)\right\rfloor
$$

Fig. 5 gives the modified MIMO channel capacities using (19) with different parameters. As we can see that the 
channel capacity of $\mathbf{H}_{i i d}$ is the largest one, which means the correlation between MIMO sub-channels will cause capacity loss. Moreover, the capacity considering MC is smaller than the one with no coupling, because the existence of MC will reduce the signal power, as well as the correlation coefficient. As for this simulation case of $d / \lambda=0.3$, the former factor is dominant.

\section{CONCLUSION}

In this paper, the effects of MC on the parameters of multiple antennas system, including signal vector, power loss, spatial correlation and channel capacity are studied. The numerical simulation results indicate that the signal powers loss is large within small inter-element distance, and the correlation coefficient reduces due to MC. Considering both power loss and spatial correlation, the channel capacity is smaller than that without MC, and the configuration of different load impedances has a very small influence on the channel capacity.

\section{ACKNOWLEDGMENT}

This work is supported by China postdoctoral science foundation (2013M541661) and fundamental research funds for the central universities (NS2015046, NS2016044), and research funds of Jiangsu Key Laboratory of Internet of Thi ngs and Control Technologies (NJ20160027).

\section{REFERENCES}

[1] Z Jie, C. Z. Gang, and H. Kikuchi, "Analysis of MIMO antenna array based on 3D von Mises fisher distribution," The Journal of China Universities of Posts and Telecommunications, vol. 22, no. 2, pp. 1523, 2015

[2] L. Zhu, Mutual Coupling Research of Multi-antenna in Dual-channel Balise, 2015.

[3] M Wang, X Ma, S Yan et al., "An auto-calibration algorithm for uniform circular array with unknown mutual coupling," IEEE Letters, on Antennas and Wireless Propagation, vol. 15, p. 1, 2015.

[4] J. W. Wallace and M. A. Jensen, "Mutual coupling in MIMO wireless systems: A rigorous network theory analysis," IEEE Trans. on Wireless Communications, vol. 3, no. 4, pp. 1317-1325, 2004.

[5] S. Lu, H. T. Hui and M. Bialkowski, "Optimizing MIMO channel capacities under the influence of antenna mutual coupling," IEEE Letters. on Antennas and Wireless Propagation, vol. 7, pp. 287-290, 2008.

[6] K. H. Chen and J. F. Kiang, "Effect of mutual coupling on the channel capacity of MIMO systems," IEEE Trans. on Vehicular Technology, pp. 1-7, 2015

[7] H. J. Song, A. Bekaryan, J. H. Schaffner et al., "Effects of mutual coupling on LTE MIMO capacity for monopole array: Comparing reverberation chamber tests and drive tests," IEEE Letters. on Antennas \& Wireless Propagation, vol 14, pp. 454-457, 2015.

[8] Z. Li, Z. Du, M. Takahashi et al., "Reducing mutual coupling of MIMO antennas with parasitic elements for mobile terminals," IEEE Trans on Antennas and Propagation, vol. 60, no. 2, pp. 473-481, 2012.

[9] J. Lee, S. Kim, and J. Jang, "Reduction of mutual coupling in planar multiple antenna by using 1D EBG and SRR structures," IEEE Trans. on Antennas and Propagation, vol. 63, no. 9, pp. 4194-4198, 2015.

[10] J. Zhou, J. Chen, and L. Qiu, "Effects of antenna correlation and mutual coupling on the performance of MIMO system in a threedimensional multipath channel," IEEE Global Symposium Millimeter Waves, pp.139-142, 2012.
[11] C. Masouros, M. Sellathurai, and T. Ratnarajah, "Large-scale MIMO transmitters in fixed physical spaces: The effect of transmit correlation and mutual coupling," IEEE Trans. on Communications, vol. 61, no. 7, pp. 2794-2804, 2013.

[12] V. G. Lykhograi, A. A. Shcherbina, V. S. Vovchenko et al., "Effect of antenna mutual coupling on MIMO channel capacity," in Proc. IEEE Conference on Antenna Theory and Techniques (ICATT) , pp. 178180, 2013.

[13] B. Clerckx, C. Craeye, and D. Vanhoenacker-Janvier, "Impact of antenna coupling on $2 \times 2$ MIMO communications," IEEE Trans. on Vehicular Technology, vol. 56, no. 3, pp. 1009-1018, 2007.

[14] Y Wu, J. W. M. Bergmans, and S Attallah, "Effects of antenna correlation and mutual coupling on the carrier frequency offset estimation in MIMO systems," in Proc. IEEE International Conference on Wireless Communications Networking and Mobile Computing, pp. 1-4, 2010.

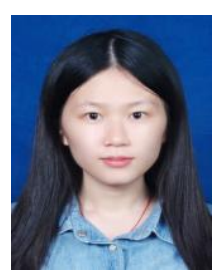

Cuiwei Xue received her BS degree in Com munication engineering from Southwest University for Nationalities, Chengdu, China, in 2014 and is currently pursuing her MS degree in electronic and communication engineering at NUAA. Her research interests include polarization channel modeling and wireless channel simulation.

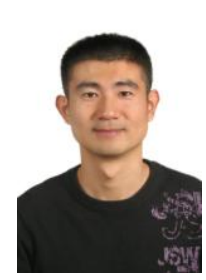

Qiuming Zhu received his BS in electronic engineering from Nanjing University of Aeronautics and Astronautics (NUAA), Nanjing, China, in 2002 and his $\mathrm{MS}$ and $\mathrm{a} \mathrm{PhD}$ in communication and information system from NUAA in 2005 and 2012, respectively. Since 2012, he has been an associate professor in the Department of Electronic and Information Engineering, NUAA. His research interests include channel modeling for next generation mobile communication, wireless channel simulation and emulator, and MIMO relay system.

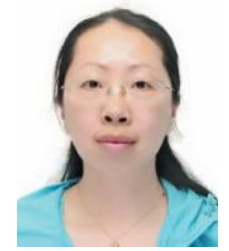

Xiaomin Chen received her BS in electronic engineering from Nanjing University of Aeronautics and Astronautics (NUAA),Nanjing,China,in 1997 and her $\mathrm{MS}$ and $\mathrm{PhD}$ in communication and information system from NUAA in2001 and 2010, respectively. Since 2010, she has been an associate professor in the Department of Electronic and Information Engineering, NUAA. Her researchinterests include adaptive technique for next generation mobile communication and MIMO relay system.

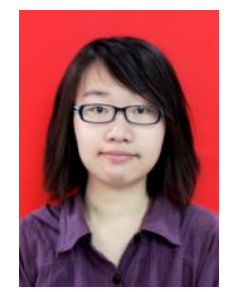

Xinglin Liu received her BS degree in communication engineering from Nanjing University of Aeronautics and Astronautics (NUAA), Nanjing, China, in 2014 and is currently pursuing her MS degree in electronic and communication engineering at NUAA. Her research interests include wireless channel simulation and MIMO channel modeling.

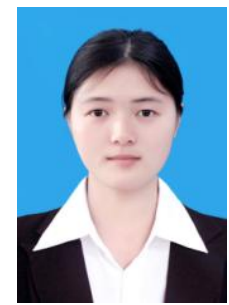

Ying Yang received her BS degree in information engineering from Nanjing University of Aeronautics and Astronautics (NUAA), Nanjing, China, in 2016 and is currently pursuing her MS degree in electronic and communication engineering at NUAA. Her research interests include polarization channel modeling and wireless channel simulation. 